\title{
Floating mirror horizon. Theory and design
}

\author{
J. Šurán̆ \\ Geodetical Observatory Pecný, Ondřejov 244, Czech Republic
}

Received November 26, 1997; accepted March 10, 1998

\begin{abstract}
A "floating mirror horizon" could substitute for the plate with mercury now used with astronomical instruments. It would have a greater and more stable reflectivity (by about 25\%) than the present surface of just mercury, and have a distinctly better definition of images. With astrolabe instruments both images of a star would have practically the same brightness. There would be no need to clean the surface of the mercury, so the particular instrument would be permanently ready for use. Theory and design of the new device are presented in this paper, along with tests from an experimental model. Regarding conditions of the mirror's flotation, it is shown that its optimum shape is that of a cylindrical plate of minimum (necessary) thickness. For some typical dimensions (with a diameter, say, of 10 or $20 \mathrm{~cm}$, and the metacentric height of the mirrors being between $20-40 \mathrm{~cm}$ ), its conditions of stability are comparable to those of transoceanic ships. The whole appliance has to consist of three basic parts: a basin with mercury; a floating mirror; and a centring device. The latter, while enabling the mirror its free floating, maintains with adequate accuracy its centring in relation to the centre of the mercury basin. This reduces to negligible (say, 0.01 ") the amount of concentric deformation by capillary forces of the surface of the mercury. It also enables preservation of proper orientation of the mirror - of its sloping line - with respect to the axis of observation and rotation of the particular instrument (of an astrolabe, PZT, etc.); and it also effectively dampens vibrations of the mirror when the instrument is rotated and set to a particular object a star, for example. In general, the surface of a floating mirror is inclined at an angle to a horizontal surface. This effect can be eliminated by simultaneous rotation of the mirror and the instrument (of an astrolabe, e.g.), or by the mirror's rotation through $180^{\circ}$ (with a PZT or meridian circle). The autocollimation method of observing a small experimental model of the floating mirror horizon showed that it possessed the expected properties.
\end{abstract}

Key words: instrumentation: miscellaneous — methods: observational - astrometry

\section{Introduction}

The horizontal surface of a fluid realizes, apparently in the most accurate way, a horizontal plane (tangent to its surface at a certain point), and is used in specialized applications with astronomical instruments: astrolabes, Photo Zenith Tubes (PZT), etc. Here, mercury in a shallow plate forms a horizontal mirror - a "mercury horizon". Its reflectivity is about $60-75 \%$ (depending on the wavelength of light), but may be somewhat lower if the surface of the mercury is not perfectly clean. Chemical reaction with some gases in the atmosphere may tarnish it; additionally, dim spots may appear over time (within an observation), reducing the mercury's reflectivity and also affecting the optical quality of images.

A higher and more stable reflectivity could be achieved with a flat mirror floating on a fluid - a "floating mirror horizon". With an aluminium coating it would reflect about $25 \%$ more incident light than the surface of mercury, and the images would have a better (sharper) definition. Its weight would render it less susceptible to vibrations; and there would be no need to clean the surface of the mercury, making the particular instrument permanently ready for observations. Using this appliance with astrolabe types of instruments, both images of a star would have practically the same brightness.

To achieve an accuracy in the definition of the horizontal (or vertical) direction, that is comparable to that of the (classical) mercury horizon, is the problem to consider. Its theoretical aspects, and possible design of the relevant device, are discussed below. Results of some trials with a small experimental model will also be given.

\section{Effects of non-plane-parallelism of floating mirror upon vertical direction}

Generally, the upper plane of a floating mirror will not be parallel to the corresponding plane tangent to the fluid, and its normal will be tilted at an angle $\Delta z$ with respect to the zenith $Z$. This situation is depicted in Fig. 1, showing 


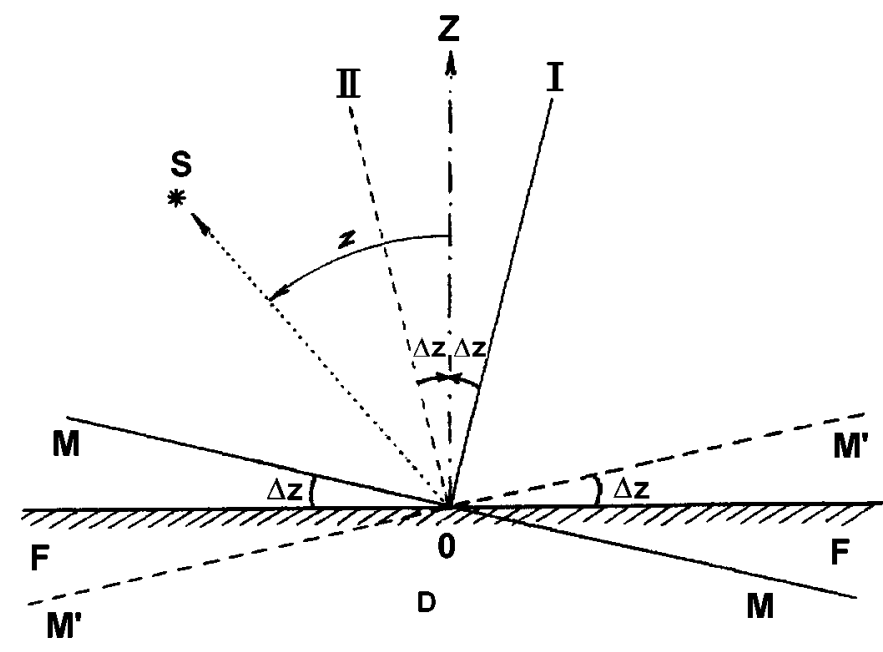

Fig. 1. Position of a floating mirror inclined by an angle $\Delta z$ with reference to zenith, and its position after rotation through $180^{\circ}$

a section of mirror $M$ on fluid $F$, perpendicular to the mirror's principal (horizontal) lines.

An object $S$ (a star) at a zenith distance $z$ with relation to the normal to the fluid $\mathrm{O} Z$ (direction to true zenith), will be observed at an angle $z+\Delta z$ with relation to the normal to the mirror $(M)$ in position I (see Fig. 1). If the mirror is rotated through $180^{\circ}\left(M^{\prime}\right)$, in position II, the angle observed to $S$ will be $z-\Delta z$. Its average value will then be $z$, and hence it will be free from any error from the tilt of the mirror to the horizontal plane.

When the mirror rotates on the surface of a fluid about $O$, its normal circumscribes the surface of a cone having the vertex angle $2 \Delta z$, whose axis is directed to the true zenith $Z$. If with prismatic astrolabes (Danjon's and the like), a floating mirror were used instead of the surface of mercury, this would revolve simultaneously with the instrument's (upper) rotatable part, and the observed zenith distances would then be $z_{0}+\Delta z=90^{\circ}-h_{0}+\Delta z=$ const., where $h_{0}$ is the instrument's altitude referred to a horizontal surface. The basic principle of the method of equal altitudes would thus be maintained. With circumzenithaltype of astrolabes the mercury's surface is stationary and centred at the instrument's rotational axis. If with these instruments the floating mirror (only) were coupled to the upper rotatable part of the instrument, and revolved with it, the above condition would also be fulfilled. If, with a $\mathrm{PZT}$, the mirror were rotated through $180^{\circ}$ (once) between exposures, the observation would likewise be referred to the true zenith, $Z$. However, generally, with regard to the family of planes perpendicular to the mirror in $O$, the deviation $\left(\Delta z^{\prime}\right)$ of the rays reflected from the mirror is not constant. This is clear from Fig. 2.

Here the (semi-) straight lines $k_{0}, k$ and $k_{\mathrm{H}}$ form a trihedron with the vertex at $O ; k_{0}$ and $k$ lie in the plane of the mirror, $k_{0}$ being a principal (horizontal) line, and $k_{\mathrm{H}}$ being produced by the intersection of a horizontal plane with

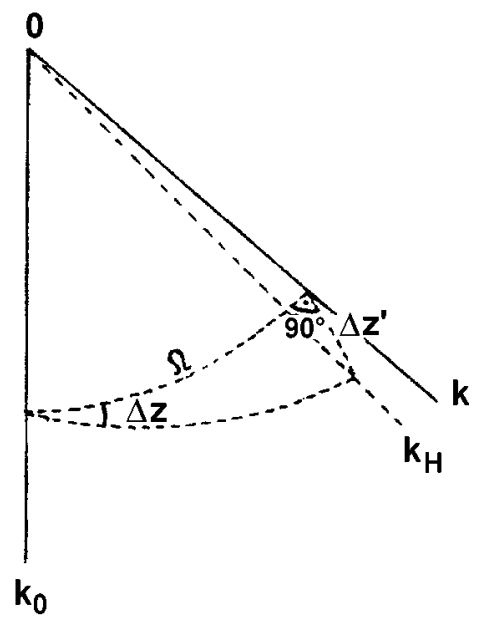

Fig. 2. Depending on angle of rotation $\Omega$, mirror's inclination $\Delta z$ is characterized by a spherical triangle

the plane perpendicular to the mirror passing through $k$. The corresponding spherical triangle to the trihedron is consequently right-angled, and the deviation $\Delta z^{\prime}$ in the direction $\Omega$ (the angle of rotation of the mirror) measured from $k_{0}$ is

$\tan \Delta z^{\prime}=\sin \Omega \cdot \tan \Delta z$.

A maximum deviation $\Delta z^{\prime}$ occurs in sections perpendicular to the mirror's principal lines (in the direction of its sloping lines, such as in Fig. 1); and $\Delta z^{\prime}=0$ in sections passing through these lines. From Eq. (1) it is also clear that the deviation in the observed zenith distance is $-\Delta z^{\prime}$ for the angle of rotation $\Omega+180^{\circ}$, as we have stated earlier.

Let us derive the error $\mathrm{d} z^{\prime}$ in $\Delta z^{\prime}$ due to variations $\mathrm{d} \Omega$ in the angle of rotation $\Omega$ (occuring from free-play in mirror's coupling with the rotational part of the instrument, or eventually, from an incorrect setting of $\Omega$ through $180^{\circ}$, e.g.). Differentiating (1), we obtain: $\mathrm{d} z^{\prime}=$ $\cos ^{2} \Delta z^{\prime} \cdot \cos \Omega \cdot \tan \Delta z \cdot \mathrm{d} \Omega$. Assuming that $\Delta z$ and $\Delta z^{\prime}$ are small quantities of the first order (this condition can be fulfilled, as will be seen later), the formula, correct to second order terms (the neglected terms being of third order and less), is:

$\mathrm{d} z^{\prime}=\Delta z \cdot \cos \Omega \cdot \mathrm{d} \Omega$.

The error $\mathrm{d} z^{\prime}$ is at its maximum, if $\Omega=0$, i.e. $\Delta z . \Delta \Omega$ (when the plane perpendicular to the mirror passes through its principal line); and it is zero if $\Omega=90^{\circ}$ (when the respective perpendicular plane is in the direction of the mirror's sloping line). From this it follows (for the error to be minimized or eliminated), that the mirror's sloping line should be oriented: (a) in the direction of the vertical plane of observation (optical axis) with astrolabe-type of instruments, (b) in an east-west direction with a PZT.

If we require that the error in zenith distance $\mathrm{d} z^{\prime} \leq$ $0.01^{\prime \prime}$, it is necessary to orient the mirror with an accuracy given in Table 1 . The values of $\Delta \Omega$ were computed here 
Table 1. Necessary accuracy in orientation of the mirror: $\Delta \Omega$ (angular); and $\overline{\Delta \Omega}$ (linear at a radius of $100 \mathrm{~mm}$ ) for some values of its inclination $\Delta z$ and certain rotation angles $\Omega$ for the error in zenith distance $\mathrm{d} z^{\prime}$ to be $\leq 0.01^{\prime \prime}$

\begin{tabular}{cccl}
$\Delta z$ & $\Omega$ & $\Delta \Omega$ & $\overline{\Delta \Omega}$ \\
\hline $60^{\prime \prime}$ & $0^{\circ}$ & $0.01^{\circ}$ & $0.02 \mathrm{~mm}$ \\
60 & 90 & 1.05 & 1.83 \\
10 & 0 & 0.06 & 0.10 \\
10 & 90 & 2.56 & 4.47 \\
\hline
\end{tabular}

according to Eq. (2), and $\overline{\Delta \Omega}$ is the corresponding linear displacement tangent to the circle of radius $R=100 \mathrm{~mm}$.

From Table 1 it is obvious that the tolerances in the mirror's orientation are moderate, if $\Omega=90^{\circ}$ (the mirror's sloping line being in the direction of observation): $\overline{\Delta \Omega}$ is then about $2 \mathrm{~mm}$ for mirror inclination $\Delta z=60^{\prime \prime}$. However, if $\Omega=0^{\circ}$, the tolerances are quite severe. The latter circumstance provides the ability to make practical adjustment of the direction of the mirror. If we first determine (by means of an autocollimation method, for instance) the mirror's position in the direction of its principal line $(\Omega=0)$, where the changes $\mathrm{d} z$ are the maximum with varying $\Omega$, we then set it into the direction of its sloping line by rotating it by $90^{\circ}$. It also therefore follows that the mirror's rotational axis has to be adequately centred. Otherwise, its fluctuations exceeding the above tolerances would also affect the accuracy of mirror's orientation.

\section{Optimum shape and balancing moment of a floating mirror - Prism-shaped floating plate}

From hydromechanics it is known that a floating body which was in a state of equilibrium, if it gets inclined, is acted upon by the balancing moment of a force couple, tending to bring it back to its original position in equilibrium. The components of this force couple are the body's weight acting in its centre of mass, and a buoyant force, acting upward in the centre of mass of the displaced liquid. The balancing moment $M$ of this force couple is

$M=W \cdot \mathcal{H} \cdot \sin \psi$,

where $W$ denotes the weight of the floating body, $\mathcal{H}$ the height of its metacentre, and $\psi$ the angle of the tilt of its axis of flotation with respect to the plumb line. The axis of flotation passes through the centre of mass of the body $C_{\mathrm{g}}$ (Fig. 3) and is vertical in the state of equilibrium when $M=0$. The metacentric height $\mathcal{H}$ is the distance from $C_{\mathrm{g}}$ to metacentre $\bar{M}$ (the position of which is given by the intersection of the plumb line passing through ${ }^{1)}$ the centre of mass of the diplaced liquid $A_{\psi}$, and ${ }^{2)}$ the axis of floating $C_{\mathrm{g}} \bar{M}$ ).

Concerning the shape of the floating body, formula (3) usually is applied to conditions of floating and stability of

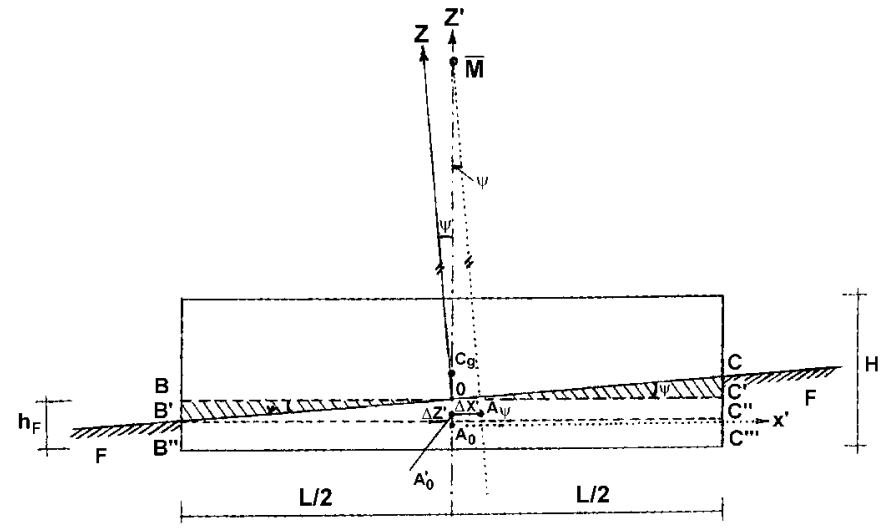

Fig. 3. An axial section of a floating plate with its centre of gravity $C_{\mathrm{g}}$, centre of gravity of the displaced fluid $A_{\psi}$ at plate's position tilted by an angle $\psi$, and its offsets $\Delta x^{\prime}, \Delta z^{\prime}$ to $A_{0}$ the centre of gravity of the displaced fluid at plate's equilibrium position; $\bar{M}$ is metacentre, and the metacentric height is the distance $C_{\mathrm{g}} \bar{M}$

ships. In our case, the floating body will have as its upper surface a plane (the mirror). Thus (unlike ships), it will be of a relatively flat shape. It is, therefore, necessary to investigate its distinguishing characteristics in relation to conditions of floating and stability - commencing with our prism-shaped plate.

Let us determine the height of the displaced liquid $h_{\mathrm{F}}$ (Fig. 3) by the floating plate in its equilibrium position. (We will not take into account the influence of atmospheric pressure: the density of the air is $0.001 \mathrm{~g} / \mathrm{cm}^{3}$.) Following from the principle of Archimedes, P.H. $\sigma=P . h_{\mathrm{F}} \cdot \sigma_{\mathrm{F}}$, and

$h_{\mathrm{F}}=\left(\sigma / \sigma_{\mathrm{F}}\right) H$,

where $\sigma$ and $\sigma_{\mathrm{F}}$ are densities of the floating plate and of fluid $F$, respectively, and $H$ is the plate's height. The relation (4) does not depend on the surface area $P$ of the plate.

In Fig. 3, there is a section through the plate in the direction of its tilt, and parallel to the plate's longitudinal edge of length $L$. In the position of equilibrium, an axis perpendicular to the plates upper surface is directed to zenith $Z$, passing through point $O$ on the surface of fluid $F$, about which the plate rotates. Point $A_{0}$, the centre of mass of the displaced liquid in the position of equilibrium, also coincides with this axis $O Z$ (plumb line). When the plate is tilted at an angle $\psi$, the centre of mass of the displaced liquid will move into the position at point $A_{\psi}$. Let us now determine the components of its offset $-\Delta x^{\prime}$, $\Delta y^{\prime}, \Delta z^{\prime}$ - with respect to a rectangular coordinate system $x^{\prime}, y^{\prime}, z^{\prime}$ having its origin in $A_{0}$ and axes parallel to the axes of the plate's symmetry. (In Fig. 3 , the axis $Y^{\prime} \equiv A_{0}$.)

The shift of the centre of mass of the displaced liquid, $\Delta x^{\prime}$ (Fig. 3), only displaces the centre of gravity of the right-angled triangle $B^{\prime} C C^{\prime \prime}$, and does not affect that of the rectangle $B^{\prime} C^{\prime \prime} C^{\prime \prime \prime} B^{\prime \prime}$ (owing to its symmetry with respect to axis $Z^{\prime}$ ). The distance of the 
centre of gravity of the triangle $B^{\prime} C C^{\prime \prime}$, from the side $C C^{\prime \prime}$ (which is parallel to the coordinate axis $X^{\prime}$ ) is $L / 3$, and that from the axis $Z^{\prime}$ equals $L / 6$. Hence, $\Delta x^{\prime}=$ $\left[P\left(B^{\prime} C C^{\prime \prime}\right) . L / 6\right] / P\left(B^{\prime} C C^{\prime \prime \prime} B^{\prime \prime}\right)$, where $P$ stands for the area of the relevant figure. $P\left(B^{\prime} C C^{\prime \prime}\right)=(\tan \psi) \cdot L^{2} / 2$, and $P\left(B^{\prime} C C^{\prime \prime \prime} B^{\prime \prime}\right)=h_{\mathrm{F}} . L$. Then for the axial section (Fig. 3),

$\overline{\Delta x}^{\prime}=(\tan \psi) \cdot L^{2} / 12 h_{\mathrm{F}}$;

and for the whole plate

$\Delta x^{\prime}=\left\{\int_{-B / 2}^{+B / 2}\left[P\left(B^{\prime} C C^{\prime \prime \prime} B^{\prime \prime}\right) \cdot(\tan \psi) \cdot L^{2} / 12 h_{\mathrm{F}}\right] \cdot \mathrm{d} Y^{\prime}\right\} / V_{\mathrm{F}}$,

where $B$ and $V_{\mathrm{F}}$ denote the plate's width and the volume of the displaced liquid. Entering $P\left(B^{\prime} C C^{\prime \prime \prime} B^{\prime \prime}\right)$ and $V_{\mathrm{F}}=$ $B . L . h_{\mathrm{F}}$ into the equation, we get $\Delta x^{\prime}=\overline{\Delta x}^{\prime}=(5)$, for we have considered a plate symmetrical to plane $X^{\prime} Z^{\prime}$; in this case $\Delta y^{\prime}=0$.

The axial shift of the centre of gravity of the displaced liquid in the direction $Z^{\prime}$ is given by

$\overline{\Delta Z^{\prime}} \quad=\left[-P\left(B O B^{\prime}\right)\left(h_{\mathrm{F}} / 2-B B^{\prime} / 3\right)\right.$

$$
\left.+P\left(O C C^{\prime}\right)\left(h_{\mathrm{F}} / 2+C C^{\prime} / 3\right)\right] / P\left(B^{\prime} C C^{\prime \prime \prime} B^{\prime \prime}\right) \text {. }
$$

Substituting corresponding expressions for areas,

$\overline{\Delta z^{\prime}}=(\tan \psi)^{2} \cdot L^{2} / 24 h_{\mathrm{F}}$.

For a plate symmetrical to plane $X^{\prime} Z^{\prime}$, it is analogously (as before) $\Delta z^{\prime}=\overline{\Delta z}^{\prime}=(6)$.

Now we can determine the metacentric height $\mathcal{H}_{\mathrm{r}}$ and the balancing moment of a prism-shaped plate $M_{\mathrm{r}}: \mathcal{H}_{\mathrm{r}}=$ $C_{\mathrm{g}} \bar{M}=A_{0}^{\prime} \bar{M}-A_{0}^{\prime} C_{\mathrm{g}} ; A_{0}^{\prime} \bar{M}=\Delta x^{\prime} / \tan \psi=L^{2} / 12 h_{\mathrm{F}} ;$ $A_{0}^{\prime} C_{\mathrm{g}}=A_{0} C_{\mathrm{g}}-\Delta z^{\prime}=\left(H-h_{\mathrm{F}}\right) / 2-(\tan \psi)^{2} \cdot L^{2} / 24 h_{\mathrm{F}}$, and

$\mathcal{H}_{\mathrm{r}}=L^{2}\left[1+(\tan \psi)^{2} / 2\right] 12 h_{\mathrm{F}}-\left(H-h_{\mathrm{F}}\right) / 2$.

At small inclination angles $(\psi)$, the second term of $(7)$ in brackets is of the 2 nd order, and the metacentric height $\mathcal{H}_{\mathrm{r}}$ is nearly constant. Body weight $W$ in Eq. (3) is for a prismshaped plate $W=$ B.L.H. $\sigma$. Taking into consideration (4), we get the following Eq. (from (3)) for the balancing moment:

$$
\begin{gathered}
M_{\mathrm{r}}=B \cdot L\left\{L^{2} \cdot \sigma_{\mathrm{F}}\left[1+(\tan \psi)^{2} / 2\right] / 12-H^{2} \cdot \sigma\left(1-\sigma / \sigma_{\mathrm{F}}\right) / 2\right\} \\
. \sin \psi .
\end{gathered}
$$

From Eq. (8) we see that the balancing moment of a prismshaped plate (in the direction of its longitudinal axis) increases with the product of its surface area $\left(P_{\mathrm{r}}=B . L\right)$, the square of its length, the fluid's density $\sigma_{\mathrm{F}}$, and the angle of inclination $\psi$. Should the balancing moment be equal also in the transverse direction, it is necessary that $L=B$, and the plate then assumes the form of a square. For the same stability in all directions, the floating plate must be cylindrical in form.

We have thus arrived at some important conclusions regarding floating conditions of the mirror, and its shape:

(1) The floating plate should be of cylindrical shape.

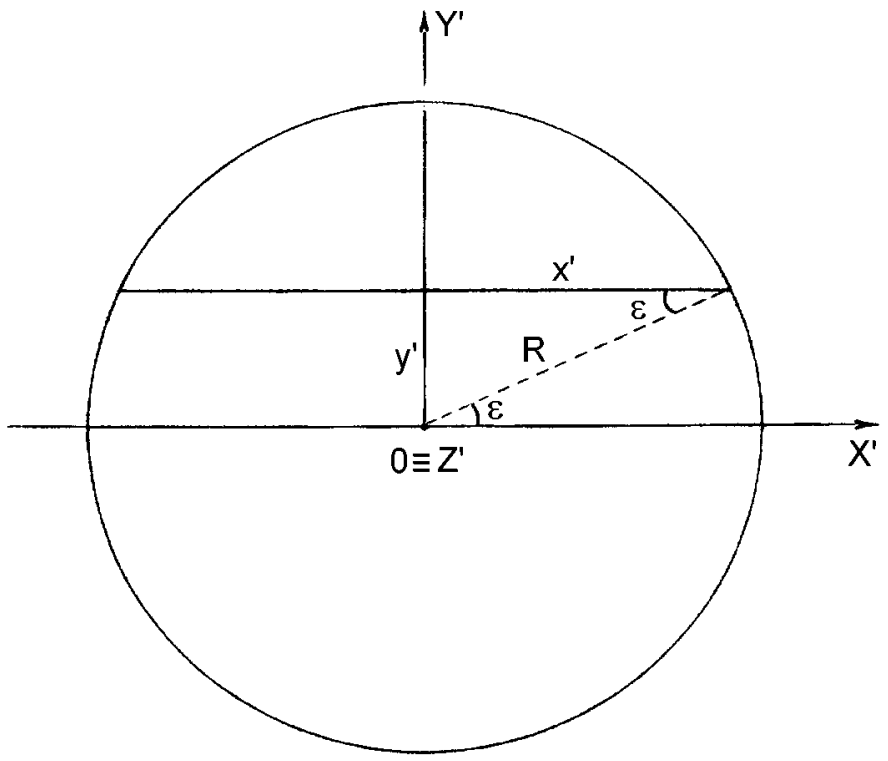

Fig. 4. Rectangular $\left(x^{\prime}, y^{\prime}, z^{\prime}\right)$ and cylindrical coordinate system $\left(R, \varepsilon, z^{\prime}\right)$ for a cylindrical plate

(2) The fluid should be of maximal density - hence mercury is suitable.

(3) The floating plate should have a minimum necessary thickness (height) and, as far as possible, should be made of a material of minimal density.

A smaller thickness diminishes the perturbing effect of the second term in Eq. (8) on $M_{\mathrm{r}}$. Theoretically, $M_{\mathrm{r}} \rightarrow \max$, if $H \rightarrow 0$ (if approaching the thinness of a floating sheet). The thickness is, however, limited by requirements of the plate's strength being sufficient to resist mechanical deformations. (With optical glass, the ratio thickness to length, or diameter, should be about 1:7.)

The cylindrical shape of the mirror also makes it possible, in conformity with a previous condition, to orient its sloping line into the required direction. (Only a circle can rotate within itself.)

Neglecting, for small inclinations, the second term in Eq. (8), the balancing moment is given by the formula

$M_{\mathrm{r}} \cong P_{\mathrm{r}}\left[L^{2} \cdot \sigma_{\mathrm{F}} / 12-H^{2} \cdot \sigma / 2 .\left(1-\sigma / \sigma_{\mathrm{F}}\right)\right] \cdot \sin \psi ;$

or still more approximately, $M_{\mathrm{r}} \approx K_{\mathrm{r}} . \psi$, since it changes almost linearly with $\psi$.

\section{Floating plate of cylindrical form}

Let us now determine the balancing moment of a floating plate of cylindrical shape with radius $R$ and height $H$; and assume a coordinate system (similar to our previous case of the prism-shaped plate), with its $Z^{\prime}$ axis coinciding with that of the cylinder (Fig. 4).

In Fig. 4, its projection onto the plane is perpendicular to axis $Z^{\prime}$. Suppose that $Z^{\prime}$ is inclined to the 
true zenith $Z$ by an angle $\psi$. For a section perpendicular to the plane $X^{\prime} Y^{\prime}$ and parallel to the coordinate axis $X^{\prime}$, we can determine the shift of its centre of gravity $\overline{\Delta x}_{\mathrm{c}}^{\prime}$ from Eq. (5), here substituting $2 x^{\prime}$ for $L$. Hence $\overline{\Delta x}_{\mathrm{c}}^{\prime}=\left(x^{\prime}\right)^{2} \cdot \tan \psi / 3 h_{\mathrm{F}}$. The total shift $\Delta x_{\mathrm{c}}^{\prime}$ of the centre of gravity of the displaced liquid in $x^{\prime}$-component then is $\Delta x_{\mathrm{c}}^{\prime}=\left[\int_{-R}^{+R} \overline{\Delta x}_{\mathrm{c}}^{\prime} \cdot P_{\mathrm{x}^{\prime}} \cdot \mathrm{d} y^{\prime}\right] / V_{\mathrm{F}}$, where $P_{\mathrm{x}^{\prime}}$ is the area of the arbitrary section. Inserting for $\overline{\Delta x}_{\mathrm{c}}^{\prime}$ and $P_{\mathrm{x}^{\prime}}=2 x^{\prime} h_{\mathrm{F}}$, $\Delta x_{\mathrm{c}}^{\prime}=\left[2 \tan \psi / 3 \int_{-R}^{+R}\left(x^{\prime}\right)^{3} \cdot \mathrm{d} y^{\prime}\right] / V_{\mathrm{F}}$.

If we use cylindrical coordinates (Fig. 4), $x^{\prime}=$ $R \cdot \cos \varepsilon, y^{\prime}=R \cdot \sin \varepsilon, \mathrm{d} y^{\prime}=R \cdot \cos \varepsilon \cdot \mathrm{d} \varepsilon$, and the numerator of the last expression for $\Delta x_{\mathrm{c}}^{\prime}$ becomes $2 R^{4} \cdot \tan \psi / 3 \int_{-\pi / 2}^{+\pi / 2}(\cos \varepsilon)^{4} \cdot \mathrm{d} \varepsilon=\Pi \cdot R^{4} \cdot \tan \psi / 4$. As $V_{\mathrm{F}}=$ П. $R^{2} \cdot h_{\mathrm{F}}$,

$\Delta x^{\prime}=R^{2} \cdot \tan \psi / 4 h_{\mathrm{F}}$.

Analogously, we get from Eq. (6), $\overline{\Delta z}_{\mathrm{c}}^{\prime}=$ $\left(x^{\prime}\right)^{2} \cdot(\tan \psi)^{2} / 6 h_{\mathrm{F}}$, and $\Delta z_{\mathrm{c}}^{\prime}=\left[\int_{-R}^{+R} \overline{\Delta z}_{\mathrm{c}}^{\prime} \cdot P_{\mathrm{x}^{\prime}} \cdot \mathrm{d} y^{\prime}\right] / V_{\mathrm{F}}=$ $\left[(\tan \psi)^{2} / 3 \int_{-R}^{+R}\left(x^{\prime}\right)^{3} \cdot \mathrm{d} y^{\prime}\right] / V_{\mathrm{F}}$. After integration, the numerator is $\Pi \cdot R^{4} \cdot(\tan \psi)^{2} / 8$ and

$\Delta z_{\mathrm{c}}^{\prime}=R^{2} \cdot(\tan \psi)^{2} / 8 h_{\mathrm{F}}$.

Owing to symmetry of the cylindrical plate with respect to the plane $X^{\prime} Z^{\prime}, \Delta y^{\prime}=0$. Further, $A_{\mathrm{c}}^{\prime} \bar{M}=\Delta x_{\mathrm{c}}^{\prime} / \tan \psi=$ $R^{2} / 4 h_{\mathrm{F}} ; A_{\mathrm{c}}^{\prime} C_{\mathrm{g}}=\left(H-h_{\mathrm{F}}\right) / 2-R^{2} \cdot(\tan \psi)^{2} / 8 h_{\mathrm{F}}$, and the metacentric height

$\mathcal{H}_{\mathrm{c}}=R^{2}\left[1+1 / 2(\tan \psi)^{2}\right] / 4 h_{\mathrm{F}}-1 / 2\left(H-h_{\mathrm{F}}\right)$.

Because $W=\Pi \cdot R^{2} . H . \sigma$, considering Eqs. (3) and (4), the balancing moment of a cylindrical plate becomes

$$
\begin{array}{r}
M_{\mathrm{c}}=\Pi \cdot\left\{R^{4} \cdot \sigma_{\mathrm{F}} / 4 \cdot\left[1+1 / 2(\tan \psi)^{2}\right]\right. \\
\left.-R^{2} \cdot H^{2} \cdot \sigma / 2 \cdot\left(1-\sigma / \sigma_{\mathrm{F}}\right)\right\} \sin \psi .
\end{array}
$$

Eventually, introducing the surface area of the cylindrical plate $P_{\mathrm{c}}$, and neglecting for small inclinations $\psi$ a 2 nd order term,

$M_{\mathrm{c}} \cong P_{\mathrm{c}}\left[R^{2} \cdot \sigma_{\mathrm{F}} / 4-H^{2} \cdot \sigma / 2\left(1-\sigma / \sigma_{\mathrm{F}}\right)\right] \sin \psi \approx K_{\mathrm{c}} \cdot \psi$.

If we compare Eqs. (9) and (14), we see that the balancing moment of a cylindrical plate is smaller than that of a square-shaped plate of the same height in an approximate ratio of 0.6 to 1 .

\section{Some numerical values}

For mercury $\sigma_{\mathrm{F}} \approx 13.5 \mathrm{~g} / \mathrm{cm}^{3}$ (at $0{ }^{\circ} \mathrm{C}$ ); the most suitable material for the mirror would be crystalline glass: Zerodur or Cervit, for instance. Its $\sigma \approx 2.5 \mathrm{~g} / \mathrm{cm}^{3}$. A mirror made of this material would have very stable dimensions due to its small coefficient of thermal expansion $(<1 \mathrm{ppm})$. The height of the displaced liquid from Eq. (4) is $h_{\mathrm{F}} \cong 0.19 H \approx H / 5$. The following Table 2 contains, for illustration and comparison, the value of the metacentric height $\mathcal{H}$, and the value of the balancing moment $M$ for some typical dimensions of square and circular shapedmirrors.

From Table 2 it is evident that the balancing moment of a plate of doubled size increases by more than an order of ten (more than twenty times), and for a cylindrical plate of $20 \mathrm{~cm}$ in diameter the metacentric height becomes about $0.4 \mathrm{~m}$. With transoceanic ships the latter is given as $0.3 \mathrm{~m}$, and with battle ships this is typically $1 \mathrm{~m}$. This indicates that a mirror of this diameter (but also that of $10 \mathrm{~cm}$ ), will have good floating stability with concomitant sensitivity in re-establishing its equilibrium.

The discussed shape of the floating plate is also suitable for the shape of the mercury plate. This can then be comparatively flat and of minimum height; and the surfaces of the plate being parallel planes conform to the requirement that the mirror and a horizontal plane be parallel. ( $\Delta z$ should be sufficiently small. Made of homogeneous material, and its surfaces being exactly plane-parallel, this would automatically be achieved.)

So far in our considerations, the floating body has only been a simple plate. Could it be of some purpose to change its form by attaching an additional mass below this? Doing so would lower the the centre of mass of the whole body, increasing thereby its metacentric height, and thus the balancing moment. But it would be quite ineffective. Calculating an increase to the balancing moment from Eqs. (9) and (14) - for instance for a square, or circular plate of the above table with thickness $H=1.5$, length $L=10$, or radius $R=5(\mathrm{~cm})$, respectively: if we change $L$, or $R$, by $\Delta L=\Delta R=1 \mathrm{~mm}$, the increase in the balancing moment would be by more than 15 times greater than that due to a change of $\Delta H=1 \mathrm{~mm}$ in the plate's thickness. This means that for a comparable effect, the additional mass attached below would have to be of considerable height, compared to that of the upper plate. It is evident that it would be impractical to enlarge the balancing moment this way, if this can be achieved much more effectively with a comparatively small change to the horizontal dimensions of the upper plate. Besides, the additional lower block would increase the vertical dimension - and amount - of the fluid (and thus of the mercury basin) required for the body to float, which would be untenable. A simple plate of cylindrical form is, indeed, the optimum shape for the floating mirror horizon.

\section{Practical design of a floating mirror horizon}

The above expounded principles lead us to the conclusion that a floating mirror horizon should consist of three basic parts:

(a) a basin with mercury,

(b) a plane-parallel mirror of cylindrical shape,

(c) a centring device.

Concerning (a), the (internal) radius of the mercury basin can, on principle, be the size normally used with a particular instrument, but allowing for necessary free spacing 
Table 2. Metacentric height $\mathcal{H}$ and balancing moment $M$ for square and circular glass plates of thickness $H$, length $L$, and radius $R$, respectively, floating on mercury (of displaced height $h_{\mathrm{F}}$ ) and inclined at an angle $\psi$ to horizontal direction

\begin{tabular}{|c|c|c|c|c|c|c|c|c|}
\hline \multirow{5}{*}{$\psi$} & \multicolumn{4}{|c|}{ Square plate } & \multicolumn{4}{|c|}{ Circular plate } \\
\hline & \multicolumn{2}{|c|}{$H=1.5$} & \multicolumn{2}{|c|}{$H=3.5$} & \multicolumn{2}{|c|}{$H=1.5$} & \multicolumn{2}{|c|}{$H=3.5$} \\
\hline & \multirow{2}{*}{\multicolumn{2}{|c|}{10}} & \multicolumn{2}{|c|}{ t- } & \multicolumn{4}{|c|}{$2 R$} \\
\hline & & & \multicolumn{2}{|c|}{20} & \multicolumn{2}{|c|}{10} & \multicolumn{2}{|c|}{20} \\
\hline & $\mathcal{H}_{\mathrm{r}}$ & $\overline{M_{\mathrm{r}}}$ & $\mathcal{H}_{\mathrm{r}}$ & $M_{\mathrm{r}}$ & $\mathcal{H}_{\mathrm{c}}$ & $M_{\mathrm{c}}$ & $\mathcal{H}_{\mathrm{c}}$ & $M_{\mathrm{c}}$ \\
\hline $0.01^{\prime \prime}$ & \multirow[t]{3}{*}{29.4} & $\begin{array}{c}5.3 \\
10^{-4}\end{array}$ & \multirow[t]{3}{*}{50.0} & $\begin{array}{c}8.5 \\
10^{-3}\end{array}$ & \multirow[t]{3}{*}{21.9} & $\begin{array}{c}3.1 \\
10^{-4}\end{array}$ & \multirow[t]{3}{*}{37.1} & $\begin{array}{c}5.0 \\
10^{-3}\end{array}$ \\
\hline 1.00 & & $\begin{array}{c}5.3 \\
10^{-2}\end{array}$ & & 0.85 & & $\begin{array}{c}3.1 \\
10^{-2}\end{array}$ & & 0.50 \\
\hline 0.00 & & 0.53 & & 8.5 & & 0.31 & & 5.0 \\
\hline$h_{\mathrm{F}} \mathrm{mm}$ & \multicolumn{2}{|c|}{$\frac{1}{2.8}$} & \multicolumn{2}{|c|}{6.5} & \multicolumn{2}{|c|}{2.8} & \multicolumn{2}{|c|}{6.5} \\
\hline
\end{tabular}

between the mirror and the basin's rim. The mirror's diameter should conform to requirement $2 R \simeq D$, where $D$ is the diameter of the objective lens. With the flat bottom of the basin extending to the vertical side of its surrounding rim, the free spacing from the edge of the mirror to the basin's rim should be about $5 \mathrm{~cm}$. As a consequence, the diameter of the basin should be $10 \mathrm{~cm}$ larger than that of the mirror. This is necessary in order to diminish the influence of deformation by capillary forces of the mercury's surface (Nušl \& Frič 1903), acting at the rim of the basin (which affects a mirror's levelled position, if the mirror is not exactly centred).

A minimal height for the mercury column below the bottom of the mirror is desirable. (An optimal height is the layer at which mercury covers the bottom surface of the basin completely. The depth is about $0.5 \mathrm{~mm}$, if there is a sublayer of amalgam. Mercury forms amalgam in contact with certain metals - electrolytical copper, silver, gold and platinum. Silver, gold and platinum can be coated as a thin layer on another material - such as steel or glass - that will not form amalgam with mercury. If the basin were made of the latter material alone, the height of the mercury column would increase significantly.) A higher mercury column would increase vibrations of mercury when the mirror was rotated or tilted.

The centring device will centre the mirror within the mercury basin, damping its vibrations at the same time, before getting into its state of equilibrium; however, it must allow for a free floating of the mirror. A centring accuracy of the mirror of about $0.01-0.02 \mathrm{~mm}$ is required, to limit the influence of the mentioned capillary effects to an anticipated $0.01-0.02^{\prime \prime}$. (The above mentioned authors, applying a differential equation establishing conditions of equilibrium between the capillary and gravity forces, derived that at a distance of, e.g., 5 and $7 \mathrm{~cm}$ from the internal wall of the basin, the deviation of a perpendicular to the mercury surface with respect to a vertical line is $<0.6^{\prime \prime}$ and $<0.01^{\prime \prime}$, respectively.) A setup of the whole appliance, conforming to requirements on prismatic as- (c)

(b)
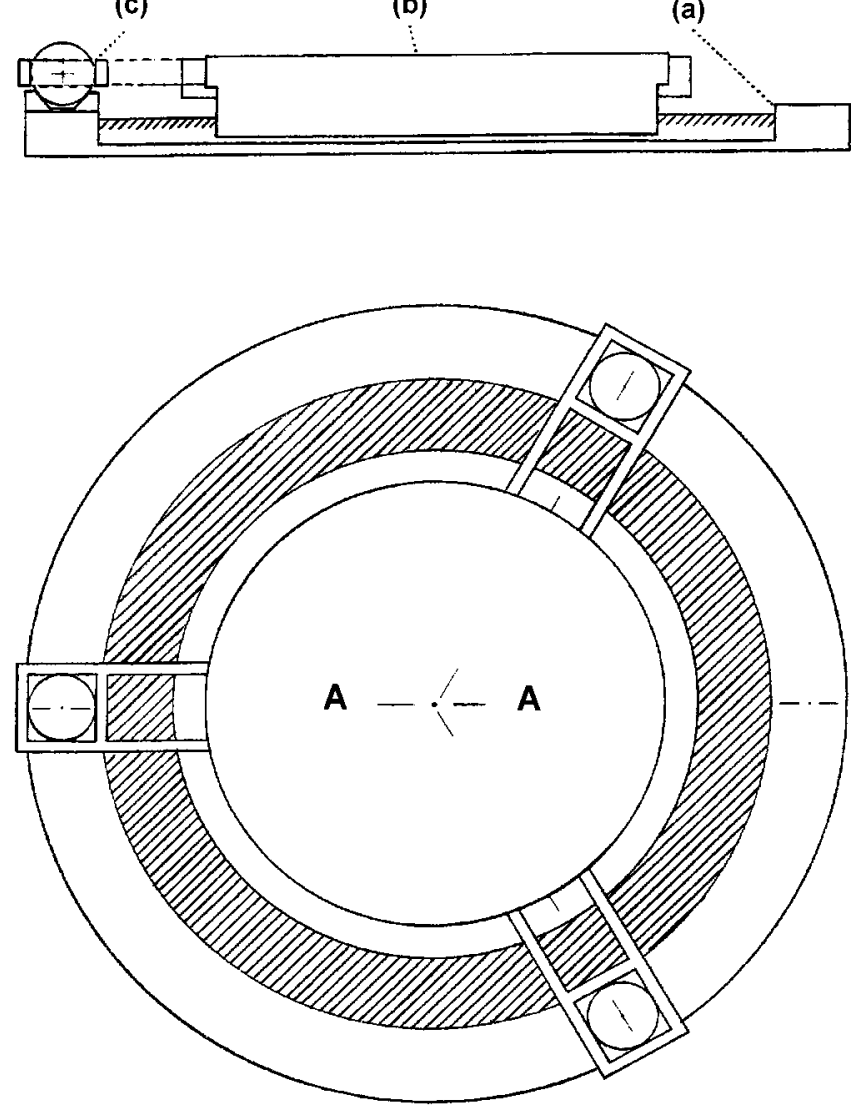

Fig. 5. Possible design of a "floating mirror horizon" (plan and Sect. A-A); (a) is the mercury basin, (b) is the floating mirror, (c) is the centring device - consisting of centring arms, fastening ring and centring balls in three fixed conical bearings

trolabes, is shown in Fig. 5 (representing a plan and Sect. A-A).

The centring device (c) is part of a fixed support extending outside the basin with mercury, and the whole appliance would rotate with the instrument. Other kinds of instruments would require modifications. With a PZT, or a meridian circle, the centring device would have to 
rest on a rotatable support - separate from the basin (a) and fitted with a bearing - to enable its rotation (with the mercury basin being stationary) through $180^{\circ}$. With a circumzenithal astrolabe, the centring device would have to be fixed symmetrically to the circular opening for the mirror and to the baseplate of its upper rotatable part (carrying the optical system), either on the upper surface of the baseplate, or beneath this. These modifications would ensure, with all instruments, proper orientation of the floating mirror (b) in the required direction.

The centring device consists of three identical blocks, with their centres situated at equal radii, and at angles of $120^{\circ}$, to the axis of rotation. At the centre of each block there is a bearing with a steel ball. The centring of the mirror is then achieved by limiting its movement relative to the three steel balls at the centres of the bearings by points of contact with the mirror's centring arms. The points of contact are tangent points of vertically oriented sides of the arms with the balls. The clearance between two sides of a centring arm and a ball is the (above) centring tolerance of $0.01-0.02 \mathrm{~mm}$, which allows for free floating of the mirror. The arms are connected to the mirror by a ring. In this the mirror rests by its extended rim, and is fastened. (No fastening is indicated in Fig. 5. Three partially recessed and loose segments inside the ring, with fastening screws attached to its outside face, could be used; and care would have to be taken to avoid imposing an excessive strain when the segments are fastened to the mirror.) If the fasten is released, the mirror can rotate freely, and its sloping line can be set to the required direction. The arms - and the connecting ring - should be made of light, homogeneous material inert to mercury and its vapours.

The centring balls (Fig. 5) rest freely in fixed bearings, having the form of a reversed truncated cone, with its sides at an angle of $45^{\circ}$ to the vertical. Upon impact by a centring arm, if the instrument is rotated, the centring balls would elevate, spiralling up from their lowest position, thus absorbing part of the impact energy; then they would oscillate until coming to rest.

In order to estimate their most effective size, we can make use of the following relations, neglecting friction in the bearings, and assuming that the first impact is only with one ball: $E_{\mathrm{P}}=E_{\mathrm{R}}+E_{\mathrm{T}}$, where $E_{\mathrm{P}}$ is potential energy of a ball, and $E_{\mathrm{R}}, E_{\mathrm{T}}$, are rotational and translational energies of the mirror. $E_{\mathrm{P}}=m . g . \Delta h$, where $m$ is the mass of the ball, $g$ is acceleration due to gravity and $\Delta h$ denotes the ball's rise above its lowest (equilibrium) point; $E_{\mathrm{R}}=1 / 2 \Theta \cdot \omega^{2} ; \Theta=1 / 2 G \cdot R^{2}$ is the moment of inertia of the mirror; $\omega$ is its angular rotational velocity; $G$, and $R$, are the mirror's mass and radius; and $E_{\mathrm{T}}=1 / 2 G \cdot v^{2}$, where $v$ is the mirror's translational velocity. The mass of the ball is open to choice with regard to its allowable free path in the conical bearing (say, $<2 \mathrm{~mm}$ ). Neglecting $E_{\mathrm{T}}$ (the motion is mainly rotational), for a typical rotational velocity of the instrument about its vertical axis of 0.5 $\operatorname{rad~s}^{-1}$ (during its setting to a star, for example), with earlier constant $\sigma=2.5 \mathrm{~g} \mathrm{~cm}^{-3}$, for mirrors with diameters of 10 and $20 \mathrm{~cm}$ (of the previous thicknesses of 1.5 and $3.5 \mathrm{~cm}$ ), and $\Delta h$ of $2 \mathrm{~mm}$ and $1 \mathrm{~mm}$ respectively, we obtain the following orientative values of diameters of steel balls $\left(\sigma=8.0 \mathrm{~g} \mathrm{~cm}^{-3}\right): 8.2$ and $10.4 \mathrm{~mm}$ for a mirror of $2 R=10 \mathrm{~cm}$, and 27.6 and $34.7 \mathrm{~mm}$ for a mirror of $2 R=20 \mathrm{~cm}$. This shows that a diameter of $10 \mathrm{~mm}$ would be suitable in the first case, and one of $30 \mathrm{~mm}$ in the second.

The described gravitational damping of the mirror's oscillations could be combined with oil-damping, if the ball bearings were enclosed in small containers which could be filled with oil (of a suitable kind) to a sufficient level. Within a symmetrical design, the oil-induced adhesive forces should not (theoretically) disturb significantly the position of the mirror's equilibrium. But the functionality of such a system (and whether it would be of any advantage) would have to be tested experimentally. The symmetrical design also minimizes - indeed practically eliminates - the effects of thermal expansion of the system upon the equilibrium position of the mirror.

Another alternative is silicon oil of a suitable viscosity, which, in a thin layer, could be used (especially at fixed stations) to coat the free surface of mercury in the basin. It is inert to mercury and would diminish the mercury's evaporation. If the speed of damping of the mirror's vibrations is unimportant, the centring balls can be fixed to the bearings. They can then be of a comparatively small diameter. But it would not be suitable to fix them to the centring arms, because this would unnecessarily increase the load on the mercury.

\section{Testing of a small experimental model}

The functioning of a small experimental model of a floating mirror horizon was tested at the Geodetical Observatory Pecný in Ondřejov. The mirror had a diameter of $10.8 \mathrm{~cm}$, a thickness of $1.17 \mathrm{~cm}$, and the steel balls in conical bearings had a $11.9 \mathrm{~mm}$ diameter, with a clearance to the centring arms of $0.02 \mathrm{~mm}$. The centring arms were made of duralumin and the basin for mercury was of stainless steel. As mercury could not form amalgam with the latter material, a minimum height of a layer of mercury completely covering the bottom of the whole basin was $1.75 \mathrm{~mm}$. This height, below the bottom surface of the mirror, was maintained for all trials. (Total height of the mercury column was $3.0 \mathrm{~mm}$ ). Trials have shown that the tested model has the expected properties. If the mirror was rotated, oscillating at a frequency of approximately $1.4 \mathrm{~Hz}$, it achieved its equilibrium position in approximately $12 \mathrm{~s}$. The frequency of oscillations (observed as oscillations of a reflected image in a telescope) was about the same as that of a freely oscillating surface of the mercury when the mirror was removed (and when the basin with mercury was rotated instead of the mirror). However, 
in this case, the free surface of the mercury did not come to rest until after about $40 \mathrm{~s}$. This illustrates the effectiveness of the damping by the new system. When the steel balls were fixed in their bearings, the reflected image still had the previous frequency of oscillations, but the system came to rest after about $17-18 \mathrm{~s}$; which is almost a $50 \%$ increase on the time interval with free balls.

Consistency of the balanced (levelled) positions of the mirror was assessed by an autocollimation method: by means of a Wild T3 universal theodolite, zenith distances of the telescope's diaphragm reflected from the mirror were observed. Multiple observations of the mirror's re-established vertical position at the zenith distance of $30^{\circ}-$ and in various directions - indicated an accuracy $\geq 0.1^{\prime \prime}$. (Accuracy of a single measure was typically $0.3^{\prime \prime}$ ). However, with a higher angular resolution of readings, the actual accuracy of the mirror, being consistently levelled, could be still higher, approaching that of the levelled (free) surface of mercury. As there are no horizontal force components acting on the mirror in its static equilibrium, no friction force to oppose the mirror's balancing moment $M_{\mathrm{c}}$ should arise between the centring arms and the steel balls (when these are in contact). Moreover, the contact is minimized to points (theoretically).

Also, if the instrument were rotated by a motor to follow stars during observation, the mirror's levelled position would not be affected when the velocity of rotation became constant. The velocity of rotation is then so low $\left(\omega \leq 7.2910^{-5} \mathrm{rad} \mathrm{s}^{-1}\right)$, that even if the motor were stopped, upon impact of the centring arms on the balls, the balls would (using previous relations) elevate by $\Delta h=2.410^{-8} \mathrm{~mm}$ with a mirror of $2 R=10 \mathrm{~cm}$ and $H=1.5 \mathrm{~cm}$, with balls having a diameter of $10 \mathrm{~mm}$; and by $\Delta h=3.310^{-8} \mathrm{~mm}$ with a mirror of $2 R=20 \mathrm{~cm}$ and $H=3.5 \mathrm{~cm}$ and a diameter of balls of $30 \mathrm{~mm}$. This means - considering that there is also an additive effect of friction in the conical bearings - that the system would behave as if the centring balls were fixed in their position, as though it were a static system in equilibrium. Because of the mentioned clearance of $0.02 \mathrm{~mm}$, within this limit the mirror has some freedom for translational motion. But this would also not affect the levelled position of the mirror, as we mentioned before.

To verify the limiting accuracy of the mirror's levelling would require a method of higher resolution (autocollimation on a meridian circle instrument, e.g.), or prolonged observations to stars with the device installed in an astronomical instrument.

A comparison was also made between the quality of images reflected from the mirror and those from the free surface of mercury. The cross-wires reflected in the autocollimation method from the mirror were distinctly sharper than those reflected from mercury, although very clean (polarographic) mercury of high chemical purity was used in the experiments. For the properties discussed, the new device could find some applications in astronomical instruments. It could also be installed in a new model of a circumzenithal.

Acknowledgements. The author wishes to express his appreciation to colleagues G. Karský and V. Skoupý, from the Geodetical Observatory Pecný in Ondřejov, for their assistance in testing the experimental model of a floating mirror horizon, as well as to A. Müller, F. Sedláček and J. Vlášek from the mechanical workshop of the Research Institute of Geodesy, Topography and Cartography in (Prague) Zdiby, who made the device according to author's proposal.

\section{References}

Nušl Fr., Frič J.J., 1903, Étude sur l'appareil circumzénithal, Bull. Int. de l'Acad. des Sci. de Bohême, Prague, 4 